\title{
Ueber die Vereinigung der Tabes dorsalis mit Erkrankungen des Herzens und der Gefäße.
}

Nebst Bemerkungen zur allgemeinen Pathologie der Tabes.

Von Prof. Dr. Adolf Strümpell in Breslau.

Im Jahre 1879 haben 0 . Berger und 0 . Rosenbach ${ }^{1}$ ) hier in Breslau zum ersten Male auf das verhältnismäßjig häufige Vorkommen einer "Koinzidenz von Tabes dorsalis und Insuffizienz der Aortaklappen" aufmerksam gemacht. Die genannten Beobachter erwähnen sieben Fälle von ausgesprochener Tabes, die mit "exquisiter Schlußunfähigkeit der Aortaklappen" verbunden waren. Weitere Betrachtungen darüber, "welche von den beiden Krankheiten die Basis für die andere abgibt“, stellen sie nicht an. Sie betonen einfach die Tatsache des häufigen Zusammentreffens „zweier so fern liegender Affektionen". 
Die Vereinigung dieser beiden Krankheiten mußte $\mathrm{zu}$ einer Zeit, wo man über die wahre Ursache der Tabes noch völlig im unklaren war, sehr seltsam erscheinen. Es ist daher nicht verwunderlich, dal man einerseits recht abenteuerliche Hypothesen aufstellte, um diesen Zusammenhang begreiflich zu machen, anderseits geneigt war, das Zusammentreffen der genannten Krankheiten überhaupt nur als etwas Zufälliges zu betrachten. Einige Autoren glaubten, die Tabes als die stets primäre Erkrankung hinstellen zu müssen, und betrachteten die Aorteninsuffizienz als eine sekundäre, trophische Stc̈rung, etwa analog den tabischen Gelenkerkrankungen, Hautaffektionen und dergleichen. Grasset stellte die Vermutung auf, die tabischen Schmerzen, d. h. die Reizzustände in den sensiblen Nerven, sollten auf reflektorischem Wege die krankhafte Störung des Herzens hervorrufen. Andere Beobachter schienen geneigt, umgekehrt die Herzerkrankung für das primäre Moment anzusehen und das Auftreten der begleitenden Tabes in Beziehung zu der bestehenden Arteriosklerose zu setzen. Noch andere Autoren hielten endlich, wie gesagt, das Zusammentreffen der beiden Erkrankungen überhaupt nur für ein rein zufälliges Ereignis. Diese Anschauung vertrat namentlich Leyden, der zuerst 1885 in seiner Bearbeitung der Tabes in Eulenburgs Real-Enzyklopädie auf die Frage zu sprechen kam. Leyden mubte auf Grund eigener und fremder Beobachtungen ${ }^{1}$ ) zwar die Häufigkeit des Zusammentreffens von Tabes und Herzfehlern anerkennen, erklärte sogar, daß er selbst die Zahl der hierher gehörigen Beobachtungen "um ein nicht unbeträchtliches Quantum vermehren könne" dem ,nicht wohl möglich sei, einen inneren Zusammenhang zwischen Klappenfehlern und Rückenmarkskrankheit anzunehmen". Auf diesem Standpunkt verharrte Leyden auch in späteren Publikationen ${ }^{2}$ ), und ihm schlossen sich Grödel u. a. an.

Ein wesentlich anderes Gesicht erhielt die ganze Frage, nachdem sich die Erkenntnis von der Bedeutung der Syphilis als der eigentlichen Ursache der Tabes immer mehr Bahn gebrochen hatte, zumal da man etwa gleichzeitig auch die große Häufigkeit der echt syphilitischen Herz- und Gefäßerkrankungen erkannte. Es lag nahe, das vereinigte Vorkommen der beiden anscheinend so verschiedenartigen Erkrankungen nicht mehr durch die unwahrschei i iche Annahme einer gegenseitigen Abhängigkeit voneinander itl eiklären, sondern dieses Vorkommen einfach als das gleichzeitige Auftreten zweier verschiedener Folgezustände derselben Ursache, nämlich der Syphilis, aufzufassen. Diese Ansicht wurde in der Tat auch alsbald von den verschiedensten Seiten geäußert. Ich selbst habe bereits 1884 in der ersten Auflage meines Lehrbuches auf die Mög. lichkeit eines gemeinsamen syphilitischen Ursprungs der beiden Krankheitszustände hingewiesen und in den späteren Auflagen diese Art des Zusammenhanges als höchst wahrscheinlich hingestellt. In ähnlicher Woise äußerten sich später Oppenheim ${ }^{3}$ ), F. Schultze $e^{4}$ u. a.

In der Folgezeit wurde auch das Naterial zur Begründung der tatsächlichen Häufigkeit des gemeinsamen Vorkommens von Tabes und Herzerkrankungen immer umfangreicher.

Ruge und $H \ddot{t} \operatorname{ttn}$ er) fanden in 138 Fällen von Tabes zwölfmal einen Herzfehler, darunter neunmal ausgesprochene Aorteninsuffizienz. Enslin berichtete über 17 Fälle von Tabes, die mit Aortenerkrankung verbunden waren, $\mathrm{Schuste} \mathbf{r}^{2}$ f fand unter $22 \mathrm{Ta}$ besfállen drei Fälle mit Aorteninsuffizienz. Zahlreiche einzelne Beobachtungen lasse ich unerwähnt.

In der überwiegenden Mehrzahl der Beobachtungen konnte eine vorausgegangene syphilitische Infektion nachgewiesen werden, während die sonst am häufigsten zu beschuldigende Ursache für einen Herzklappenfehler, eine vorhergegangene akute Polyarthritis, nur ausnahmsweise angegeben wurde. Sehr auffallend war in allen Beobachtungen das starke Ueberwiegen der Erkrankungen an den Aortaklappen über die Mitralfehler. Im Zusammenhange hiermit mehrten sich auch die Angaben, daß die Beteiligung der Zirkulationsorgane an dem

1) Die alteren Beobachtungen findet man alle zusammengestellt in der Thése von Jaubert, Parıs 1881. - 2) Zentralblatt für klinische Medizin 1887, No. 1. 3) Deutsclie medızinische Wochenschrlft 1 1888, No. 45. - Deutsche inedizınische Wochenschrift 1888, No. 20. - Diskussion im Verein fär innere Medizin, Berlin. $1888-4)$ Sitzungsberichte der niederrheinischen Gesellschait für Heilkunde, Mal l $>92$ -5) Berliner klinische Wochenschrift 1897, No. 35. - 6) Berliner Dissertation 1898. 7) Deutsche medizinische Wochenschrift 1903 , No. 41 . tabischen Prozeb sich keineswegs allein auf die Aortaklappen beschränke, sondern daß ebenso oft die Aorta selbst in der Form starker Arteriosklerose oder insbesondere des Aortenaneurysmas erkrankt gefunden wurde. Erwähnenswert ist in dieser Hinsicht namentlich eine von F. Lusser angestellte, bei Leyden und Goldscheider (Krankheiten des Rückenmarks) zitierte Statistik, wonach in 96 Sektionsprotokollen von Tabesfällen $18 \mathrm{mal}$ ein gleichzeitiges Aneurysma der Aorta erwähnt gefunden wurde.

Diese neu gewonnene Kenntnis von der Häufigkeit der Aortensklerose und des Aortenaneurysmas bei der Tabes war zunächst in rein klinischor Hinsicht von Wichtigkeit, da sie uns ein besseres Verständnis für gewisse bei der Tabes schon lange bekannte Symptome ermöglichte. lch erinnere hier zunächst an die schon von Charcot betonte Häufigkeit des Vorkommens einer anhaltend hohen Pulsfl'equenz bei vielell Tabeskranken, eine Erscheinung, die meines Erachtens mindestens in einem großen Teil der Fälle auf die begleitende $\mathrm{Ar}$ teriosklerose zu beziehen ist, und dann vol' allem an die von v. Leyden ${ }^{1}$, Grödel u a. geschilderten Anfälle von Angina pectoris und cardialem Asthma bei Tabeskranken. Wel gegenwärtig insbesondere die von v. Leyden mitgeteilten Krankengeschichten aufmerksam liest, wird sich der Annahme kaum enthalten können, dak es sich in diesen Fällen weit wahrscheinlicher um die Symptome einer begleitenden Aorten sklerose als um „neulalgische Anfälle im Bereiche der Herznerven des Vagus" gehandelt hat. AuRer diesem klinischen Interesse trat aber die ätiologische Bedeutung des häufigen Vorkommens von Aortensklerose und Aortenaneurysma bei der Tabes immer stärker hervor, seitdem auch für diese beiden Gefäßerkrankungen die große ursächliche Bedeutung der Syphilis sicher nachgewiesen war (Hampeln, A. Helle $\mathbf{r}^{\mathrm{u}}$ u. a.). In wie anderem Lichte manche frühere Mitteilungen jetzt erscheinen, zeigt z. B. die von v. Leyden ${ }^{2}$ ) gemachte, interessante Beobachtung, daß eine 40 jährige Frau, die seit Jahren "durch die Pflege ihres an Tabes erkrankten, unbehilflichen Mannes sich stark angestrengt hatte, seit $1 / 2 \mathrm{Jahr}$ die deut. lichen Zeichen eines Aneurysmas des Arcus aortae" darbot. v. Leyden führt diese Beobachtung als Beleg für die Entstehung eines Aortenaneurysmas "durch Ueberanstrengung" an. Gegenwärtig wird man wohl kaum mehr daran zweifeln, daf eine zwischen den Eheleuten übertragene syphilitische Infektion die befriedigende Erklärung für das erwähnte Volkommen der beiden Erklankungen abgibt.

So hat sich also allmählich die Verbindungskette zwischen den drei Erkrankungen Tabes, Syphilis und Aortensklerose mit Aneurysmabildung immer fester gezogen. An die Stelle unwahrscheinlicher Hypothesen sind klare ätiologische Anscliauungen getreten, und gerade in diesem sich Ineinanderfïgen der einzelnen, scheinbar zusammenhangslosen klinischen Beobach. tungen liegt ein neuer, nicht unwichtiger Beweis für die Richtigkeit der gegenwärtig nicht mehr zu bezweifelnden Annahme von dem syphilogenen Ursprung einerseits der Tabes, anderseits vieler Fälle der genannten Gefäßerkrankungen. Allein, von der wirklich bestehenden Häufigkeit des gemeinsamen Vorkommens dieser Geschwisterkrankheiten erhält man doch erst dann eine richtige Vorstellung, wenn man auf Grund der neueren diagnostischen Fortschritte diesem gemeinsamen Auftreten der beiden Krankheitsformen seine bosondere Aufmerksamkeit zuwendet. Fast alle bisherigen, hierher gehörigen klinischen und anatomischen Untersuchungen gehen von der Tabes aus und betonen, dab gorado die weiter fortgeschlitten e tabische Erkrankung verhältnismäßig oft mit Herz und Gefäßaffektionen vereınıgt ist. Infolge einiger zuällig gemachten Beobachtungen habe ich in den letzten Jahren umgekehrt mein Augenmerk besonders darauf gerichtet, wie häufig man in Fällen unzweifelhafter Aortenerkrankung (Aortensklerose, Insuffizienz der Aortaklappen, Aneurysmen der Aorta) gleichzeitig ausgesprochen tabische Erscheinungen nachweisen kann. Diese Untersuchungen sind erst möglich geworden, seitdem die Diagnose der beiden in Betracht kom. menden Krankheitsgruppen durch die neueren Fortschritte in der Untersuchungstechnik so erheblich an Sicherheit und

1) Zentralblatt tïr kllnische Medizin 1887, No, l. - 2) Zeitschrift für klımısche Medizın Bd. XI, S. II2. 


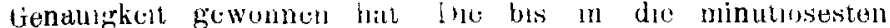
Einzelheiten durchgefuhrte llurchfor'schung le's schiel' unerschöpflıchen tabischen Symptomenkomplexes hat cine Menge nener und wichtiger dugnostischer $\Lambda$ nhaltspunkte ergeben, aus denell das Bestehen ener tabischen Erkrankung des Nervensysteins schon in ihrell frühell Antängen und ill ihlen rudimentäl'sten Fol'men mit Sicherheit elschlossen welden kiann Für die Diagnose der Aortensklerosc und des Aortenanenrysnas blldet anderseits die Röntgenuntersuchung der Brustorganc einen so enorinen Fortschritt, daB man erst jetzt überhaupt in der Lage ist, eın richtiges Urteil über die Häufigkeit und dıc Wichtıgkeit di:r Aortenerkrankungell zıl gewinnen, während dıe Erkrankungen der Aortenklappen schon durch die alteren physikalischen Untersuchungsnethoden in den meisten Fiallen mit vollster Sicherheit erkannt werden künıcn. Seitde.m ith einmal meine Aufmerksamkeit auf das Vorkommen encelner tabischer Symptome bei meinen Kranken mit Aortafehlurn gerichtet hatte, war ich orstaunt über die Hảufigkeit dieses gemeinsamen Vorkollumens. Binen groben Tell der alı memer Klinik gemlachten Beobachtıngen uber die Vereinigung roll Erkrankungen der Zirkulationsorgane mit tabischen Symptomen (24 einzelne Fialle) haben bereits meine Assistenten Rogge und $E$ Miiller veröffentlicht ${ }^{1}$. Im Vellaufe des letztcll Jihres habe ich weitele 16 hierher gehörige Fälle beob. achtet, darunter einige, die den ursprünglichen Berger Roscnbachschen Bcobachtungen entspiechen, d. h. Kranke init ausgesprochener Tabes, bei denen die Untersuchung des Herzens einell deutlichen Aortenfehler ergab. Mein besondcres Allgenmerk wandte ich aber, wie gesagt, den Herzkranken, Illil zwar besonders den Krankell mit arteriosklelotischen Her 4 beschwerden zu. Wenn man bei derartigen Kranken, dic nur ihrer Herzbeschwerden (Kurzatnijgkeit, Stenokardie, Herzklupfen, Husten, Oedenie etc) wegen den Alzt aufsuchen, sorgfaltig nach einzelnen tabischen Symptomen forscht, so ist man cl'staunt, wie häıfig man sie nachweisen kanıl. Fur die Krauken selbst haben die meisten dieser tabiselien Symptome kicine besonderc Bedeutung, denn es handelt sich nicht un schwerere ataktısche Störungen etc, sondern meist nur um die Zeichen der beginnenden, oder richtiger der sogenannten rudinıentären Tabes (s. u.).

Zu beachtell ist zunächst stets das Verhaltch der Pupillin. Findet man enge oder ungleiche, lichtstarre Puulllen. so ist damit die Diagnose eigentlich schon gesichert; denn wir wissen, dab die reflektorische Pupillenstarle bei nolmalcun Verhalten des Augenhintergrundes fast immer als Teilelscheinung eines tabischen Prozesses zll deuten ist. In einz..Inen Fällen war die Lichtstarre der Pupillen das einzige uberhaupt vorlaandene tabische Syınptom. In eillem Fall (durch dic Autopsio bestätigte schwere Endoaortitis, Myocarditis und Insuffızienz der Aortaklappen) war nur die rechte Pupille lichtstarr und entrondet, die linke Pupille nolmal. Ich zweifle nicht darall, dilb wir es auch hiel mit einer rudimentären tabischell Affektion zil tun hattell.

In der Regel wird man freilich neben den Pupillenverändel'ungen noch andere tabische symptome nachweisen köınen. Besondere Aulmerksamlicit verdient bekanntlich das Verhalten der Sehnenreflexe Solange man die Symptomatologie der Tabes mur nach den schon weiter fortgeschrittenen Fallen beurtelte, mubte das Felilen der Patellarreflexe als Regel erschennen. Gegenwärtig wissen wir, dab die A chillesSichnonreflexe gewöhnlich noch vol den Patcllarreflexen rerschwinden, entsplechend dem Umstande, dab die ticfer ce-

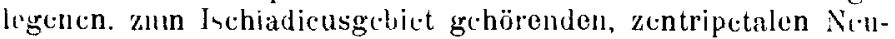
ronc meist frïher rou der krankhet belallen werden als dic höher getegenen Cruralısfascru. Demgemiib fanden auch wır in unseren fuallen wiederiolt boreits feblcude Achullesreflexc. wiblend dic Patellareflexe noch dentlich vorlanden warcil. Ji, wie auch Rogge und Mäller schon hervorgehoben habell. findet man die Patcllarreflexe sogar zuweilen gestergert, während die Achillesleflexe bereits sthr schwach sind oder ganz fehlen. Die Steigerung der Reflexe scheint in d.M T'it dor erste Allsdruck der beginnenden Elkrallkung der betreftenden Fisern zil sein, ähnlich wit die Hyperästhesle d(r An-

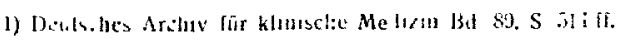

asthesie, oder die ınotolische Reizerscheinung der Lähıung vorhergchen kaın. In cinzolncn Fällen hatten wir Gelegenheit, das allmähliche Schwäclıerwerden und schlließliche Verschwinden der vorher gesteigorten Reflexe direkt zu beobachten. Wie bei den Pupillen, so kommen auch bei den Achillesund dell Patellarreflexen vereinzclt Ungleichheiten auf beiden Seiten vor.

So war z. B. bei einer 48jährigen Frau, deren Mann ebenfalls an Tabes und Hersfehler litt und die selbst cine ausgesprochene Aortenslilerose mit Herzhypertrophie darbot, der rechte Patellarroflex ge steigert, wahrend der linke kaum deutlich anslösbar war. Außerdem bestanden seit zehn Jahren typische lancinierende Schmerzen.

Neben den Sehnenreflexen verdient auch das Verhalten des Miskcltonus an den unteren Extremitäten zur Erkennung del beginnenden Tabes Beachtung. Man beurteilt den Muskeltollis nach dem Umfange der leicht und schmerzlos ausführbaren, passiven Beweginngen. Wenn man z. B. den gestreckten Oberschenkel im Hüftgelenk leicht so woit beugen kann, dab der Fub den Kopf des inı Bett liegenden Kranken berührt, oder wenn mall das Bein in Hüftgetenk mühlos bis $90^{\circ}$ abduziclen kann, oder wenn man bei Bauchlage des Patienten den Unterschenkel leicht so weit beugen kann, daß man mit dem Hacken den Glutäus tief eindrückt, so darf mall sicher von einer Hypotonie der betreffenden Muskelı sprechen. Anl besten ist es, die passiven Bewegungen bei dem Kranken mit den entsprechenden passiven Bewegungen bei einem gleichaltrigen Gesunden zll vergleichen. Man gewinnt dann am besten ein Urteil über die normale und die krankhaft gesteigerte Dehnbarkeit der Muskeln. Notwendig ist es auch, alle Gelenke (Huifte, Knie. Fuß und Zehen) einzeln durchzuprïfell. Wie schon frülıere Beobachter (Frenkel, 0. Förster) hervorgehoben haben, fuldet man die Hypotonie oft scholl „im präataktisclıen Stadiun" der Tabes. Auch wir laben sie ziemlich häufig als tabisches Symptom bei unseren Arteriosklerotikern und Herzkranken festgestellt. Physiologisch interessant ist die Frage nach den Beziehungen der Hypotonie zu den Sehucnreflexen, da der Muskeltonus doch ebenfalls als reflektolische Erscheinung aulzufassen ist. Die Erlahrung hat uns gezeigt, dal die beiden Symptome - Abnahme der Sehnenreflexe und Abnahme des Muskeltonus - nicht parallel laufen. Wir haben Faille mit gesteigerten Patellarreflexen und gleichzeitig deutlich ausgesplochcner Hypotonie gesehen. Dabei ist helvolzuheben, dap mall Tomlus und ruflcktorisches Verhalten stets an demselben Muskel initeinander vergleichen muß!

Auf die übrigen bekannten Initialerscheinungen der Tabes brauche ich hier nicht näher einzugehen. Kurz erwähnen will ich nul' die Notwendigkeit einel genauen Sensibilitätsprüfung an der Haut des Rumpfes zur Allfindung der bekannten gürtelförmigen hypästhetischen Zonen und endlich die Notwendigkeit einer genauen Analluese zur Feststellnng typischer lancinielender Schmerzen, Blasellstörungen, etwaiger gastrischel Krisen u. dgl. Je solgsamer man in dieser Hinsicht verfähıt, um so häufiger wird mall zu positiven Ergebrissen kommen. Natürlich muß aber bei der Deutung aller Angaben nuit der nötigen Vorsicht und Umsicht verfahren werden.

Die allgenıine pathologische Bedcutuıg des verhältnisInäßjir häufigen Nachweises von mehı oder weniger zahlreichen tabischen Sylnptomen bei kranken mit "arteriosklerotischen“ Beschwerden sehe ich, wie schon erwähnt, vor allem in dem hierin liegenden. crneuten Hinweis auf die ätıologische Bedeutung der Sypliilis sowoll-für dic Entstchung von Herz- und Gefaßßklanklıeitcn, als auch anderseits fül die Entstehung der Tabes. Delın nur unter dicser Voraussctzung konnen wir ein bcl'riedigendes Ver'ständıis für dıeses häufige Zusammenvorkonımen der beiden Kranklıeiten grivinuen. Es war uns auch möglich, in der groben Mehrzahl der Fälle init Bestimmtheit oder wenigstens mit groficl. Walurscheinlichkett dell Nachwcis der vorhergegangenen syphılitischeı Infelition zu führen. Rogge und Müller fandell in ihrell 24 Fällen neuninal sichcre Syphilis, zehnulal höchst wahrscheinlich eine vorausgegangene luftektion. Im ganzen war also in $79 \%$ der Fälle Syphilis mit der größten Wahrscheinlichkeit als d:e Ersache der GefäBcrkraukung und der gleichzeitigen Tabes zu betlachten. Unter menen neucren 16 Filllen war zelnmal, d. $i$ in $62 " \%$, Syphlis 
mit Sicherheit oder grolser Wahrscheinlichkeit vorlaanden. doch auch in den iibrigen sechs Fälen warell Verdachtsgriunde verschiedener Art nacliweisbar. Ich persïnlich neige mich. je mehr meine Erfahrungen wachsen, immormehrder Ansichtzu, dabdio Syplitis iiberhaupt die eiuzige wesentliche Ursache, d. h. die Conditio sine qua non der Tabes is t. Fül die Gefäßerkrankungen gilt dieser Satz natiirlich keineswegs, da sicher Schädlichkeiten verschiedener A rt zu chronischer Endarteriitis (Arteriosklerose), Endo-Aortitis und Endocarditis der Aortaklappen mit ihren Folgezuständen führen können. Findet man aber in einem derartigen $F$ all gleichzeitig sichere tabische Symptome, so weisen diese fast mit Bestimmtheil aucl beim Felılen sonstiger Syphiliszeichen und beim Fehlen entsprecliender anamnestischel Angaben -... auf eine frühere syphilitische Infektion und somit wenigstens nit groler Wahrscheinlichkeit auch auf die syphilitische Natıl der Herz- oder Gefäßerkrankung hin. Del sichere Nachweis e inzelner begleitender tabischer Symptome (Pıipilenstarre, fehlende Sehnenreflexe u. dgl.) hat somit dieselbe Bedentung fül den Nachweis einel vorhergegangenen Syphilis, wie etwa der Nachweis charakteristischer Narben an den Geschlechtsteilen oder den unteren Extremitäteı u dgl. Natürliclı wird man alıch alle sonstigen bedeutsamen Momente (also bei Aortafehlern insbesondere das Fehlén einer vorausgegangenen rheumatisclien Infektion, bei Aneurysmen ein relativ jugendliches Alter der Erkrankten u. dgl.) in Betracht zielien ind sein Urteil erst auf Grund aller Erwägungen abgeben. Von größiter Bedeutung wild voraussichtlich in nicht zu ferner Zeit die Sero-Liagnostik der Syphilis werden, deren schon jetzt in naher Aussicht stehendes Ziel darin besteht, die Diagnose der friiheren spezifischen Infektion ohne jede Riicksiclit auf Allamnese und noch bestehende Symptome zu stellen. Erst wenn dieses Ziel sicher erreicht ist, werden wir vollkommen ullanfechtbare $\Lambda \mathrm{n}$ gaben über die ätiologische Bedeutung der Syphilis gewiınen. Uebrigens lonnte schon jetzt in ermem unserer Fälle von GefäBerkrankung und Tabes, bei dem die Lues voin Patienten selbst völlig in Abrede gestellt wurde, auf der liesigen Ne issersclien Klinik die Serumreaktion im Blut und in der Cerebrospinalfluissigkeit mit völlig sicherem positiven Ergebnis angestellt werden.

Dio Bedeutung der Syphilis als der wesentlichon Ursache für die gemeinschaftliche Entstehung von Gefäberkrankungen und Tabes läBt von neuen die Frage auftauchen nach der besonderen Art der Entstehung dieser Folgezustionde der syphilitischen lnfektion. Schon vor Jahren habe ich die Ausicht ausgesprochien, daß wir den tabischen anatonischen Prozeb nicht in eine Linie mit den tortiären syphilitisclıen Neubildungen stellen dürfen und daß wir daher, falls wir ihn als eine direkte Folge der syphilitisclien Infektion betracliten wollch, seine Entstehung in anderer Weise deuten müssen als die Entstehung der spezifisch gummösen Prozesse. Ich faßte daher die Tabes (und ebenso die verwandte progressive Paralyse) als Wirkung der durch die Syplilis entstandenen, spezifisehen 'Tox in e auf', eine Annahme, die sich durch zahlreiche $A$ nalogien. vor allem durch das Anftreten der postdiphtherisclıen lähnın. gen und Ataxien stützen lieb. Meine Anschauung lat, wie mir scheint, vielfachen Anklang gefunden und läßt siclı auch jetzt naclı der grundlegenden Schaudinnschen Entdeckung der Syphiliserreger aufrecht erlialten, da die Spirochäten sich bekanntlich in allen echt gunmösen Neubildungen leicht nachweisen lassen, wälırend sıe bei del Tabes und der Paralyse im erkrankten Geliirn und Rückennark ebenso wenig aufzufinden sind wie etwa Diphtheriebazillen in den nach einer Diphtherie degenerierten motorischen Gaumennerven. Ich möchte jetzt die Vermutung aussprechen, daib dic ,atheromatösen" "und "sklerosierenden" Prozesse an den Gefát)wändell ınd an delı Aortaklappen, welche den "syphilitischen" Herz- und Gefäb-

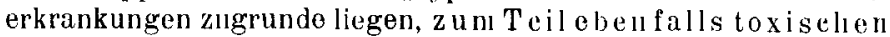
Ursprungs sind. Es gibt wohl zweifellos Erkrankungen der Aorta und des Icerzmiskels, dıo schon anatomisch als gunnnöso spezifische Neubildungen aufzulassen sind, abel 111 der Regel sind doch die anatomisehen Verïnderungen, die wir hej dell in kede stehenden Fällen an Gefäbapparat beobachten, von dem gewohnlichen "Arterienatherom" nıcht wesentlıch unter- schieden Die Arteriosklernse ist eben nicht in dent Sinne ein spezifiselier anatomischer Prowe wie etwa dio Bildung eines Tuberkelkuöteliens, einel diphtherkehen Vlemblan oder eines Glummas Die Arteriosklerose ist der anlatomischer Aısdruck der allgemeinen Schädigung, der sbuntzung. der Nekrose der Arterienintina und der übrigen defähwand. Sle kann daher durch Schïdlichkeiten verschiedenel $\Lambda \mathrm{rt}$, aber gle ichförmiger Wirkung hervorgerufen werdell, und $z u$ diesen Schädlichkeiten gehört auclı die Einwirkung der syphilitischeı Toxine. Die Aunalıme clomischer Schädlichkeiten liegt luer besonders niahe, da wil auch sonst als die lauptsächlichsten atiologischen Momente fur die Entstehung der Arteriosklerose chemische Einfliïsse anuehınen (Alkolıol, Tabak, gichtischo Diathese u. a.) nnd da auch die experimentellen Studien ijber die Arterienerkrankıng durch Adrenalineinwirkung auf dellselben Entstelungsmodus hinweiscn. Selbstrel'ständlich kamı jede der besonderen Sclsädlichkeiten trotz der im allgemeinen gleichlartigen Wirkung auch ihre besonderen charakteristischell Eigentuimlichkeiten zeigen in bezug anf I,okalisation, Intensität und Eigenart der Erkrankung So bietet auch die syphilitisclic $\Lambda$ rteriosklerose dem geubten Auge des erfahrenen pathologischen Anatomen gewisse Eigenloiten dal," und vol allem ist ihre Vereinigung mit echt gummösen Prozessen ebenso leicht möglich und ebenso häufig vorhanden wie nach neueren Erfahrungen die nicht seltene Vereinigung del Tabes mit eclit gummösen meningitischen Veländeruıgen.

Der so allgemein ausgesprochene Gedanke von der grund. sätzlıchen Verschiedenheit der direkton Spirochätenwirkung (Bildıng der syplilitischen Neubildung) und der metasy philitischen Toxiuwirkungen (Tabes, Paralyse, Arteriosklerose) ist natïrlich erst der Ausgangspunkt fur zahlreiche weitere noch nötige Untersuchungen Die bosoudere Natur der 'l'oune, ihre Entstehıng, die Alt ihrer Einwirkung - dies alles bedarl noch del Elforschung. Trotzden ist auch der allgemeine Gedanke schon nicht ganz wertlos, weil er uns eiı Verständnis für manche sonst nllerklärliche Tatsachen elmögliclit. so vor allenı fïr di* eigentümliche CesetzinäBigkeit in der anatomischen Lokilisation des tabisclien Prozessos, und ferner fuir den anllallenden Gegensatz in der Wirkung der spezifischen antisyphilitischen Arzneimittel (.) Jod und Quecksılber) einerseits bei den gummösen P'rdukten der Syplitilis und anderseits bei den toxischen metasyphilitischen Degenerationsprozessen. Ienn wenn jch auch die Anwendung von Jod ind Quecksilbor bei der Behandlung der Tabes keineswegs fül ganz bedentungslos erklairen will, so wird mir doch jeder Al'zt darin beistimmen, daß die Wirkungen dieser Mittel bei der Tabes und der Paralyse nicht entfernt dieselben sind wic etwa bei den tertären Haut-, Sclıleinıhaut- ınd Periosterkiankungen, deı gumıösen Erkraıknıgen der Gelirnarterien 11. a. Etwas Achnliches beoback tet man aucl in bezug auf die metasyphilitischen Gefäberkrankung('n I)ie oft gerïhmte Hellwirkung des Jods bei arteriosklerotisclien Elkrankungen hängt zwar vielleicht init der syphilitischen Natıl eines Teils der Erkrankmugsfälle zusammen; diese Heilwirkung ist jedoch übcrlatupt nur verlialtnismäbig selten sehr deutlich ausgesprochen. Sicher fohlt sie oft anch in solclen Fillen. deren syphilitisclıer Uısprıng dırch sonstige Unstäıdo höchst wahl'sclieinlich ist.

Fasse ich somit dic häutige Verbindung von scliwerer Arteriosklerose und arteriosklerotischen Herzelkrankungen nit tabischen Symptomen als die gleichzeitige Folge derselben. oder nindestens nahe verwand ter tox ischer. metasyplilitischer Schädlichkeiten auf, so entsteht dir Frage, ob noch weitere begleitende Unistände den Auftreten dieser besonderen Folgezustände Vorschub leisten. la Tabes. Paralyse und Arteriosklerose kemeswegs in jeden Falle vou syphilis aufteten, so mulf thre Entstehung doch noch von besonderen, nicht immer vorhandenen Unständell ablıängig seirl. Insofern diese Umstände ill der besonderen Art der Infektion. etwa in der spezifjschen Qualität Vurulenz) der Infektionserreger liegen. entzichen sio sich einstweilen noch völlig enner nälıeren Betrachtıng. Etwas anssichtsreicher erschent dagegen die Ueberlegung der Fragen, inwieweit die besonderen indıviduellen Verhältnisse des von der Infektion belallenen Organismus von Einflub aluf die weiteren Wirkungen drescre lufektion sind und inwleweit noch andere begleitende äuBero Nebollumiställde in Betracht kommen. Die 
Entstehung aller exogenen, d. h. auf äll Bero Schädigungen | zurückzuführenden Krankheiten, können wir auf die allgemeıne Formel $K=\underset{W}{S}$ zurïckfuhren. wobei $K$ die Krankheit, $S$ die krankmachende Schädlıchkeit und $W$ den ihr rom Organismus geleisteten Widerstand bedeutet. Nur wenn W klcincr als $\mathrm{S}$ ist, kann die Krankheit wirklich ontstehen, sic wird um so schwerer, je größer $S$ und je kleiner $W$ ist. Nun sctzt sich aber $S$ oft zusammen aus der eigentlichen wesentlichen, spezifıschen Krankheitsursache, der conditio sine qua non (z. B. der syphilitischen Infektion bei der Tabes), und gewissen begleiten den Schädlichkeiten (Trauma, Ueberanstrengung, psychische Errregungszustände, Erkältungen, diätetische und andersartige toxısche Schädlichkeiten). Statt des einfaghen $S$ können wir also $S_{w}+S_{b}$ selzcn, deren Summe bci kleinem $S_{w}$ doch geniigend grob ist, wenn nur $S_{b}$ einen hohen Wert hat, und umgekehrt. Der Summand $S_{W}$ mub immer vorhanden sein, $S_{b}$ kann gelegentlich fehlen. Auch der individuelle Widorstand (W) des Organismus setzt sich zusammen aus $\mathrm{z}$ wei Faktoren: aus seiner angeborenon individuell-konstitntionellen Widerstandskraft ( $W_{a}$ ), die aber durch spätere, im Leben erworbcne und auf sie einwirkende linflüsse $\left(W_{e}\right)$ wach sen (durch zweckmäBige Lebensweise, gute Ernährıng, Muskelübung, künstliche linmunisierung u. a.) oder (durch unzweckmäßige Lebensweise, schlechte Ernährung, toxische Einflüsse u. a.) abnehmen kann.

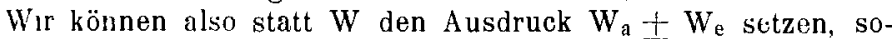
daß also unsere Gesamtformel für die Entstehung exogener Krankhoiten $K=\frac{S_{w}+S_{b}}{W_{a}+W_{e}}$ lautet. Dabei kann es für einzelne schädliche Faktoren freilich zweifelhaft bleiben, ob sie zur Vergrößer.ung von $S_{b}$ oder von $W_{e}$ mit negativem Vorzeichen beitragen. Der Erfolg bleibt natürlich derselbe.

Fïr dıє Tabes und die boigeordneten Erkrankungcn des Gefäbapparats ist die Syphilis die wesentliche Conditio sine qua 110n. In bezug auf die Tabes sind begleitende Schädlıchkeiten, dio $S_{b}$ oder $W_{e}$ beeinflussen, im allgemeinen selten nachweisbar. Nur Traumen spielen eine gewisse Rolle. Aber der Hauptsach nach scheint doch der spezifische Enfluß des $S_{w}$ in Betracht $z u$ kommen. $O b$ und wieweit diesem $S_{w}$ noch ein individueller Widerstand $W_{a}$ entgegenwirkt, entzieht sich einstweilen noch unserer Kenntnis. In bezing auf die inetasyphilitische (und vielleicht auch die spezifisch syphilitische) Gefäberkrankıng spielon aber meines Erachtens die begleitenden Umstände oine nicht Innerhebliche Rolle. Die leichte Angreifbarkeit der Arterienintima ermöglicht, wie wir bereits erwähnt haben, eine nicht geringe Verschiedenartigkeit der zur Wirksamkeit gelangenden Schädlichkeiten und, wie in manchen anderen Fällen, so scheint auch hier gerade die Summation mehrerer Schädlichkeiten oft von besonders nachteiliger Wirkung zu sein. Die Ellahrung lehrt uns daher, daß in den Fällen von metasyphilitischer Geläßerkrankung verhältnismäßig oft gleichzcitig auch sonstige schädigende Faktoren nachweisbar sind, die vielleicht 111 andercn Fällen schon für sich allein zu einer Erkrankung der Gëfaßwand fuhren können. Ich denke hier besonders an une dauernde körperliche Ueberanstrengnng, an chronischen Alkoholismus und vor allem an die schädlichen Folgen des anhaltendell, ubermäßigen Rauchens. Es ist vielleicht nicht Zufall, dall gcrade hier in Breslau zum erstenınal die hänfige Vereinigung der Tabes mıt Herz- und Gefäßerkrankmugen gefunden ist und daß ich selbst auch erst hier in Breslau auf die grobo Häufigkeit dieser Vereinigung aufmurksam guworden bin Sie crklärt sich, wie ich meine, vielluicht zum Teil durch don bei uns im Osten so sthr verbreiteten übermäßigen Zigarr(11- und Zigarettengenufi. Jedenfalls glaube ich, zum Teil auf diešn Umstand die auffallendc Häutigkeit der arteriosklerutıschen Holz- und Gefäßerkrankungen in unseren östlichen Gegenden zurückfihl’en zu können. Alkoholismus, schwerc Arbeit, hereditäru Veranlagung kommen ebenfalls oft genug in Betracht. Doch nöchte ich inmerbin betonen, daß man nebon dicsen Beyleitumstanden doch stets der wesentlichen Krankheitsulsache das größte Gewicht beilegen soll. Oft genug ist von begleitenden Momenten nichts nachwershar, so dak also nur das wesculliche ursächliche lloment (die Syphilis nnd die metasyphilitische Intoxikation) in Betracht kommt.
A uf die neucrdings, namentlich von Fidinger betonte Bedentung der funktionellc:n Schädignng für die Entstehung dernervöscn Degencrationszustände (Tabes u a.) $\left.{ }^{1}\right)$ möchte ich hier nicht näher eingehen. Es wal ein guter Gedanke von Edinger, darauf hinzuweisen, daß dio Funktion selbst mit einer gewissen Schädigung des funktionierenden Gowebes verbunden ist, die nur durch erneuten Gewebsersatz ausgoglichon werden kann. Ich glaube aber, daß Edinger in der Heran. ziohung dieses Vorgangs zur Erklärung pathologischer Verhältnisse zu weit gegangen ist und zu manchen etwas künstlichen Deutungen seinc Zuflucht nehmen muß, um seine Theorie allgemein durchzuführen. Nur in einer Hinsicht habe ich selbst schon vor Edingor die funktionelle Schädigung, d. h. den Verbrauch von Organmasse durch die Organfunktion, zur Aufklärung der Aetiologie der Tabes herangezogen. Es bedarf nämlich der Erklärnng, warum die angenommene metasyphilitische, toxische Schädigung so häufig zu einer vielo Jahrc und selbst dahrzehnte lang fortschreitenden Erkrankung führt. Bei den zum Vergleich herangezogenen toxischen Degenerationszuständen nach anderen Infektionskrankheiten (Diphtherie u. a.) handelt es sich um ziemlich akute Degenerationszustände, die entweder zum Tode führen, oder zur Heilung kommen, oder viclleicht zu schlieflich stationaren Ausfallserscheinungen führen. Bei der Tabes (und der analogen progressiven Paralyse) handelt es sich aber in vielen Fällen um eine stetig progressive, auf immer neue Nervengebiete übergreifende Krankheit. Diosen Vorgang habe ich dadurch zu erklären versucht (Lehrbuch, VIII. Aufl. 1894, Bd. III, S. 236), daß ich die andauernde Funktion des einmal toxisch geschädigten Gewebes fül die schließliche Degeneration desselben mit verantwortlich niachte. Der Funktion würde hiernach zwar eine wichtige, aber doch nur sekundäre Bedeutung zukommen. Die eigentliche conditio sine qua non ist meines Erachtens die spezıfische toxische Schädigung. Sie allein erklärt die merkwïrdige elektive Lokalisation des tabischen Krankheitsprozesses, die dem gesamten Krankheitsbilde der Tabes sein so äußerst charakteristisches und trotz aller $\mathrm{Ab}$ weichungen im einzelnen doch so merkwürdig gleichförmiges Gepräge gibt. Erwähnen möchte ich hier noch kurz, daf die bei der Tabes vorkommenden, höchst eigenartigen „Krisen“ (vor allem die "gastrischen Krisen“, ferner die Kehlkopfkrisen, die Schmerzanfällo etc.) ebenfalls am besten durch die Annahme spezifischer toxischer Schädlichkeiten erklärt werden können. Hier müssen freilich noch besondere, unbekannto Verhältnisse im Ablauf des Infektionsprozesses in Betracht kommen, die aber der späteren genaueren Erforschung wohl zugänglich sein dürften. Diese Verhältnisse müssen besonderer Art sein, da bekanntlich viele Tabesfälle ohne alle "Krisen“ verlaufen.

Bereitet, wie ich soeben angedeutet habe, der chronischprogressive Verlauf der Tabes dem Verständnis gewisse, größtenteils $110 \mathrm{ch} \mathrm{zu}$ überwindende Schwierigkeiten, so erscheint es dagegen um so leichter begreiflich, daß die Tabes in sehr vielen Fallen diesen progressiven Verlauf nicht zeigt. Wie so oft in der Entwicklung der klinischen Pathologie, trat auch das Krankheitsbild der Tabes seinen Entdeckern zuerst nur in voller Entwicklung und Ausbildung als scharf abgegrenzte Krankheitseinheit vor Augen. Viel später erst lernte man auch schon aus den ersten Anfängen den kommenden, schweren Krankheitsprozeb voraussagen, und das Suchen nach den Hilfsmitteln für die Diagnose der "beginnenden Tabes" hat lange Zeit die Neurologie beschäftigt. Jetzt wissen wir aber, daß nicht jede „begrinnende Tabes“ sich wirklich weiter zu dem Symptomenkomplex der voll entwickelten Tabes weiterbildet, daß dıe anfänglichen Symptome in vielen Fällen stationär bleiben, ohne daß neuc Erscheinungen hinzutreten. Wir müssen also von der „initialen Tabes“ die „rudimentäre Tabes“ wohl untersclieiden, obwohl natürlich diese Unterscheidung keine vollkommen strcnge sein kann und stets mehr eine retrospektive als ene prognostischo Bedeutung hat. Wenn wir Tabesfälle beotachten, wo die Symptome auch nach 10-15jährigem und läıgtrom Bestehen immer nur noch etwa in Pupillenstarre. fehlenden Patellarreflexen und gelegentlichen. leichten lanciuierenden Schmerzen bestehen, ohne dab sich eines der

1) Deutsche medizınische Wochenschrıft 1904, No. 45. 
schweren tabischen Symptome (Ataxie etc.) hinzugesellt hat, so können wir wohl mit weit mehr Recht von einer rudimentären, d. h. in ihrer Entwicklung stehen geblicbenen. als von einer beginnenden Krankheit sprechen.

Gerade die von mir bei cluronischen Herz und.Geläberkrankungen beobachteten Fälle gelı̈ren nun zum nicht geringen Teil zur rudimentären Tabes. Es haben sich nur einzelne tabische Symptome entwickelt die dauerno bestehen bleiben, sich aber nicht weiter vermehren Darum treten sie auch nicht besonders stark hervor und werden oft von den viel wichtigeren cardialen und vaskulären Krankheitserscheilungen fast ganz überdeckt. Man muß besonders nach ihnen suchen. Dann findet man sie leicht und ist ïberrascht von ihrer Häufigkeit.

Bei diesen rudimentären Tabessymptomen handelt es sich also um gewisse Ausfallserscheinungen, bedingt durch toxische Degenerationen gewisser Nervenbahnen, die an sich abgeschlossen und keiner weiteren Aenderung mehr fällig sind. Auch bei dem Fortschreiten des begleitenden Herz- und Gefäßleidens braucht man nicht immer eine fortdauernde Einwirkung der metasyphilitischen Intoxikation anzunehmen, da bei den besonderen, hier obwaltenden Verhältnissen schon die einmalige Schädigung eine andauernde Erschwerung und schliebliche Störung des Kreislaufs nach sich ziehen kann.

Von Wichtigkeit scheint mir zu sein, daß sich ähnliche Verhältnisse, wie ich sie in bezug auf die Vereinigung von Herz- und Gefäßerkrankungen mit tabischen Symptomen hier ausfïhrlicher erörtert habe, auch sonst noch nachweisen lassen. Die fast völlige Analogie der progressiven Paralyse mit der Tabes habe ich schon wiederholt betont. Das oben Gesagte gilt daher ganz ebenso für die in gleicher Weise bestehenden Beziehungen zwischen der Paralyse und den begleitenden Erkrankungen des Gefäßapparates. Ich selbst verfüge über mehrere, hierher gehörige klinische Beobachtungen. Anderseits sind aber auch die syphilitischen Erkrankungen des Gefä̉apparates nisht die einzigen, die sich gelegentlich mit tabischen Symptomen vereinigen. Auch bei der Syphilis anderer innerer Organe (der Leber, des Darmes etc.) muß man meines Erachtens stets an die Möglichkeit dieser Kombination denken. So kann z. B., wie ich gesehen habe, die syphilitische Natur einer sonst ihrer Art nach zweifelhaften Lebererkrankung durch das Auffinden einer gleichzoitigen Pupillenstarre erkannt werden. Jedenfalls ist in atiologisch zweifelhaften Fällen der Nachweis einer rudimentären tabischen oder paralytischen Er. krankung stcts eirl diagnostisch wichtiges Moment, welches eincn wertvollen Hinweis auf die besondere Natur der Erkrankung gibt. Ebenso wie man trotz des Fehlens bestimmter anamnestischer Angaben aus dem Vorhandensein charakteristischer Narben, aus dem Nachweise früherer häufiger Aborte u. dgl. oft genug die frühere Syphilis nachweisen kann, mub man auch in jedem der Lues verdächtigen Falle sorgfältig nach einzelnen tabischen Symptomen suchen. Ihr Nachweis kann in manchen Fällen die sonst nicht $z$ u treffende Entscheidung ermöglichen.

Resümoe. 1. Bei Kranken mit Insuffizienz der Aurtaklappen, Sklerose der Aoita und Aortenaneurysma findet man bei besonders daranf gerichteter Auimerksamkeit nicht selten Zeichen einer wleichzeitigen tabischen Eıkrankung (reflektorische Pupillenstarre, fehlende Selınenreflexe, lancinierende Schmerzen il a.).

2. Anderseits vereinıt sich die aungesprochene Tabes dorsalis vorhaltnısmäßig häufıg mit Aorteninsuffizienz und Aortasklerose.

3. Diese Kombination der beiden Erkrankungen beruht darauf, daß beıde denselben Ursprung haben. Sie sind Nachkrankheiterı einer vorhergegungenen syphilitischen Infektion

4 Der Nachweis einzelner tabischer Symptome, ror allem der reflektorischen Pupillenstarre, kanu überhaupt als Nachweis eines syphlitischen Stigmas betrachtet werden und ist somut von becou. derer diagnost ischer Bedeutung. 